\title{
Efecto del plasma rico en plaquetas en la regeneración ósea posexodoncia del tercer molar impactado evaluado a través de tomografía computarizada Cone Beam
}

\author{
Effect of platelet-rich plasma on alveolar bone repair following impacted \\ third molar surgery evaluated to Cone Beam computerized tomography
}

\author{
Dilmer A. Quincho-Rosales ${ }^{1,2}$,Teresa Evaristo-Chiyong ${ }^{1,3}$, Marina G. Portocarrero-Gallardo ${ }^{1,4}$, \\ Dalia V. Churampi-Mancilla ${ }^{5}$, Sergio Chui-Galván ${ }^{6}$, Sixto Grados-Pomarino ${ }^{1,7}$
}

\section{Resumen}

Introducción: El plasma rico en plaquetas (PRP) es un biomaterial con alta concentración de plaquetas y factores de crecimiento que favorecen la regeneración de tejidos tras su aplicación en intervenciones quirúrgicas como la exodoncia del tercer molar. Material y métodos: El estudio se realizó con 20 pacientes con necesidad de exodoncia del tercer molar inferior bilateral. Las exodoncias se realizaron en un mismo tiempo quirúrgico y en uno de ellos, escogido aleatoriamente, se aplicó el gel de PRP y el otro tomado como grupo control. La evaluación posoperatoria se llevó acabo posterior a los tres meses; los parámetros evaluados fueron: dolor posoperatorio, curación de herida y densidad ósea mediante tomografía computarizada Cone Beam. Resultados: El dolor posoperatorio durante el tercer día, primera y segunda semana presentó menor grado en los sitios de aplicación de PRP que el grupo control ( $\mathrm{p}<0,05$. La densidad ósea medida por la tomografía computarizada Cone Beam evidenció ser mayor en los sitios de aplicación de PRP que el grupo control $(\mathrm{p}<0,05)$. Conclusión: El PRP posee efecto en la disminución del dolor posoperatorio y aceleración en la cicatrización de herida. Además evidencia mayor densidad ósea en comparación con la no aplicación del PRP.

Palabras clave: Plasma rico en plaquetas, tercer molar, regeneración ósea.

\section{Abstract}

Introduction: Platelet-rich plasma (PRP) is a biomaterial with a high platelets concentration and growth factors enhance tissue regeneration after application in surgical procedures, such as third molar extraction. Material and methods: The study was performed with 20 patients with indication for third bilateral lower molar extraction. The extraction were performed in the same surgical time and one of them the PRP gel was applied (randomly chose) and the other as control group. The postoperative evaluation was carried out until three and a half months, the parameters evaluated were: postoperative pain and wound and bone density using Cone Beam computed tomography. Results: Postoperative pain during the third day, first and second week was lower grade than sites of PRP application to the control group $(\mathrm{p}<0.05)$. The bone density measured by Cone Beam CT was higher at the sites of PRP application than the control group $(\mathrm{p}<0.05)$. Conclusion: PRP has an effect in reducing postoperative pain and accelerating wound healing, and it also shows higher bone density compared to not applying PRP.

Keywords: Plaleth rich plasma, thrid molar, bone healing.

${ }^{1}$ Universidad Nacional Mayor de San Marcos, Facultad de Odontología. Lima, Perú. ${ }^{2}$ ORCID 0000-0003-1344-0542. ${ }^{1,3}$ ORCID 00000001-7129-5617. ${ }^{1,4}$ ORCID 0000-0002-2871-1846. ${ }^{5}$ Universidad Nacional Mayor de San Marcos, Facultad de Ciencias Biológicas. Lima, Perú. ORCID 0000-0003-374. 'Hospital Nacional Hipólito Unanue, Servicio de Radiología Bucal y Maxilofacial Lima, Perú. ORCID 0000-0001-6161-2420. ${ }^{1,7}$ ORCID 0000-0002-4404-5230. 


\section{Introducción}

La cirugía del tercer molar conlleva recuperación posoperatoria prolongada, siendo un reto para el cirujano el manejo clínico de la cicatrización de heridas, principalmente, por la respuesta inflamatoria de los tejidos $\operatorname{afectados}^{(1,2)}$. El alveolo posexodoncia cicatriza por segunda intención, reparando o regenerando el tejido óseo alveolar, tal proceso implica una prolongada recuperación posoperatoria ${ }^{(3)}$. La aceleración del proceso de reparación estructural del tejido blando y óseo afectado, promueve la reducción de complicaciones posoperatorias; por ello, la búsqueda de biomateriales aplicados en heridas quirúrgicas, con la finalidad de acelerar el proceso de cicatrización, es una tendencia para el manejo óptimo de las intervenciones quirúrgicas ${ }^{(4,5)}$.

En la actualidad existen diversos biomateriales con capacidad en regeneración tisular, de estos, la literatura actual resalta el uso de biomateriales autólogos (provenientes del mismo individuo) como el plasma rico en plaquetas (PRP) ${ }^{(6)}$. El PRP es un concentrado plaquetario (seis a siete veces el número basal) rico en proteínas denominadas factores de crecimiento (FC), con propiedades en la aceleración de cicatrización de heridas y regenerativas ${ }^{(7)}$. El mecanismo de acción se fundamenta en los FC que actúan como mediadores bioquímicos capaces de modificar las respuestas celulares, como proliferación y diferenciación, de las células diana (células madre mesenquimales, osteoblastos, fibroblastos, etc.), promoviendo la rápida respuesta fisiológica de cicatrización y, en gran medida, promoviendo la regeneración tisular ${ }^{(8)}$.

La aplicación del PRP sobre heridas quirúrgicas, muestra un consenso sobre las ventajas que posee, principalmente, por disminuir el tiempo de cicatrización; sin embargo, es todavía cuestionable su capacidad regenerativa, específicamente, sobre el alveolo posexodoncia ${ }^{(9)}$. Investigaciones recientes resaltan las bondades del uso del PRP éste, evidenciando la mejoría clínica posoperatoria (disminución del dolor, menor grado de inflamación, bajo riesgo de complicaciones, etc.), asumiendo tal mejoría a una rápida cicatrización ósea. Sin embargo, gran parte de la evidencia pone énfasis en resultados más concretos como análisis radiológicos e histológicos, afianzado los resultados sobre regeneración ósea ${ }^{(5,10,11)}$.

El uso masivo del PRP como regenerador óseo, supone la exhaustividad de los ensayos clínicos para llegar a consensos sobre su aplicación. Por ello, la necesidad de estudios bien estructurados es importante para determinar su uso de manera convencional. Las investigaciones difieren, principalmente, por el método de obtención y el tipo de estudio, dificultando así la evidencia sostenible ${ }^{(12)}$.
El PRP es versátil en su modo de obtención, además brinda gran ventaja sobre los demás biomateriales, sobre todo, por la fácil reproductibilidad de los protocolos más usados ${ }^{(13)}$. Asimismo, la versatilidad del PRP también presenta desventaja debido a que no se puede consensuar los estudios en base a un solo protocolo. A pesar de esta dificultad, la mayoría de estudios se centran en el efecto clínico del PRP más que el modo de obtención ${ }^{(3)}$.

La falta de consenso sobre el uso del PRP en la cirugía del tercer molar pone en relevancia la propuesta de evaluar el efecto que posee el PRP sobre la regeneración ósea posexodoncia evaluada mediante la tomografía computarizada Cone Beam (TCCB) y el consecuente beneficio clínico que conlleva su uso.

\section{Material y métodos}

\section{Pacientes}

El presente ensayo clínico, a simple ciego, se llevó acabo en la clínica de Cirugía Bucal y Maxilofacial (CBM) de la Facultad de Odontología de la Universidad Nacional Mayor de San Marcos (FO-UNMSM). El estudio contó con la participación de pacientes que acudieron a la clínica de CBM, con requerimiento de exodoncia del tercer molar mandibular bilateral. La selección de los participantes en la investigación se determinó mediante lo siguiente:

\section{Criterios de inclusión}

1. Pacientes con edades de entre 18 a 30 años de ambos sexos.

2. Pacientes con requerimiento de exodoncia del tercer molar mandibular bilateral en condiciones similares de complejidad para la exodoncia (Figura 2).

3. Pacientes ASAI.

4. Estar de acuerdo con el consentimiento informado.

\section{Criterios de exclusión}

1. Tercer molar con pericoronaritis o infección local activa.

2. Piezas dentarias incluidas o con diferencias, entre estas, en el grado de complejidad para la exodoncia.

3. Pacientes con discrasias sanguíneas.

4. Pacientes con deficiencias metabólicas.

5. Pacientes fumadores

6. No estar de acuerdo con el consentimiento informado.

Al obtener la historia clínica del participante apto, se les explicó los objetivos del estudio con detalles del 
procedimiento y el tiempo requerido, plasmado en el consentimiento informado.

\section{Obtención del PRP}

El protocolo de obtención del PRP se basó en los estudios de Antonello ${ }^{(10)}$ y Anitua ${ }^{(5)}$, estandarizado en el laboratorio de Citología y Genética Humana de la Facultad de Ciencias Biológicas de la UNMSM; cuyo indicador fundamental fue obtener el número plaquetario, por ciclo de centrifugación, superior a 6 veces del conteo basal.

Se extrajo $16.2 \mathrm{ml}$ de sangre venosa periférica de la fosa anterocubital en 6 tubos de $2.7 \mathrm{ml}$ con anticoagulante citrato de sodio al 3.8\% (tubo celeste), y $6 \mathrm{ml}$ de sangre en tubo sin anticoagulante (tubo rojo), (BD Vacutainer ${ }^{\circledR}$ Franklin Lares, NJ USA). Los tubos celeste se centrifugaron a 1235 RPM y
FCR de 185g (GEMMY-PCL3) durante 10 minutos; en paralelo, se adicionó $1.5 \mathrm{ml}$ de gluconato de calcio al $10 \%$ (Laboratorio Sanderson S.A.) al tubo rojo y se mantuvo en reposo, también, por 10 minutos. Pasado el tiempo, al tubo rojo, se agitó vigorosamente por unos segundos e inmediatamente después, se centrifugó por 3 minutos a una velocidad de 3266 RPM y FCR de $1288 \mathrm{~g}$, mientras a los tubos celeste ya centrifugados se les extrajo con micropipeta el tercio inferior de la fracción plasmática, correspondiente a la porción rica en plaquetas, en un recipiente de vidrio estéril. Finalizada la centrifugación del tubo rojo la fracción plasmática superior obtenida corresponde a la trombina autógena. Finalmente, para la obtención del gel de PRP se mezcla el plasma del tubo celeste con la solución del tubo rojo a razón de 4:1, la gelificación se llevó acabo de entre 1 a 2 minutos después de la mezcla (Figura $1)$.

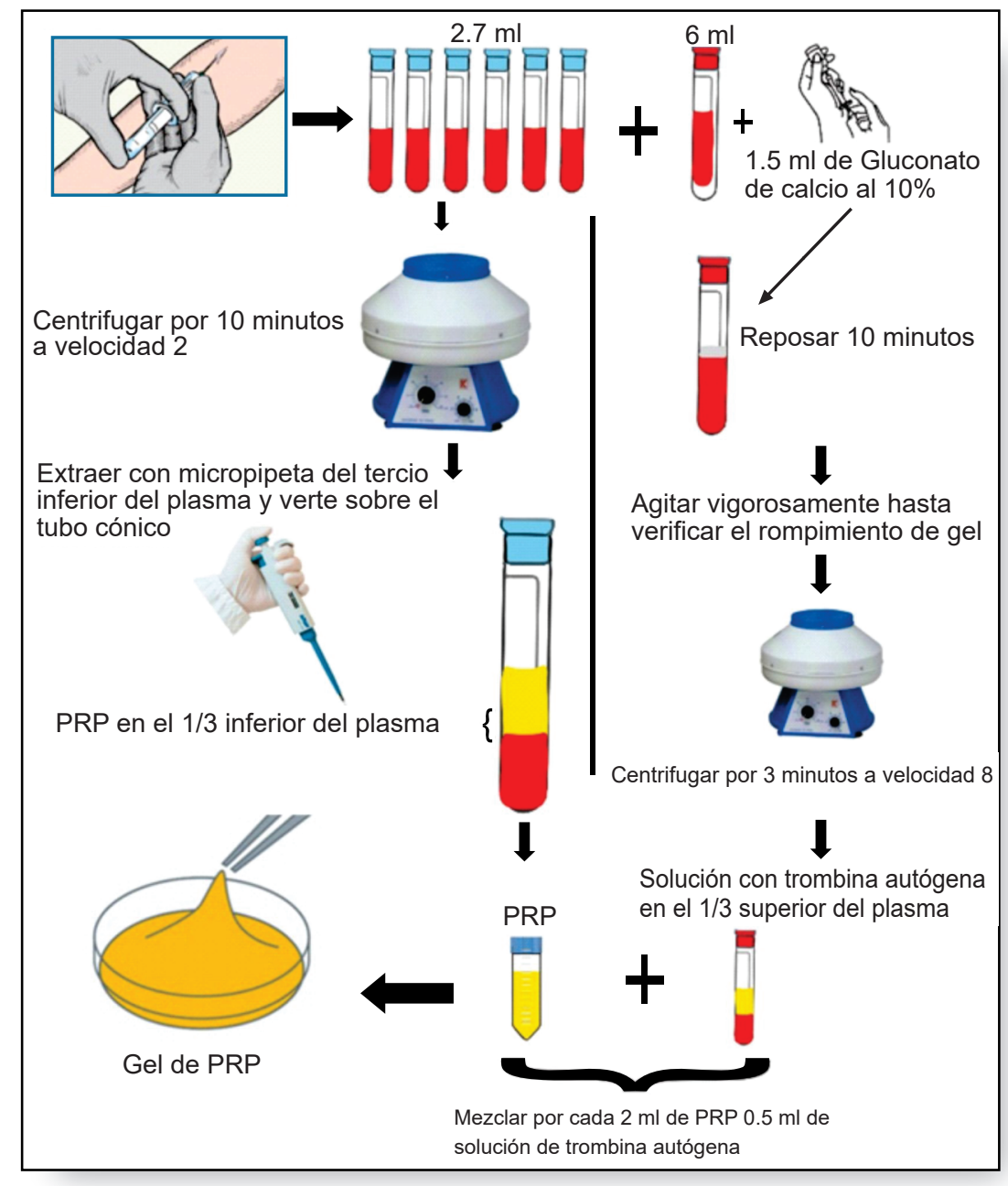

Figura 1. Esquema de protocolo de obtención del PRP. Velocidad 2 (1235 RPM y FCR de 185g), velocidad 8 (3266 RPM y FCR de 1288g). 


\section{Procedimiento quirúrgico y aplicación de PRP}

El procedimiento quirúrgico se realizó por especialistas y residentes de CBM. Se realizaron las exodoncias de las piezas 38 y 48 en un mismo tiempo quirúrgico, conformando en un mismo paciente el grupo control y experimental; sobre un alveolo posexodoncia se aplicó el gel de PRP más sutura (grupo experimental), y sobre el otro solo sutura (grupo control); la elección se determinó por aleatoriedad. La medicación analgésica y antibiótica posquirúrgica fue la misma para todos los casos (Figura 2).

\section{Evaluación posquirúrgica}

Los pacientes fueron evaluados al tercer, séptimo y catorceavo día para control posquirúrgico y retiro de puntos de sutura (séptimo día); asimismo, se evaluó el dolor posoperatorio según la escala visual analógica (EVA) y nivel de cicatrización de herida según los criterios propuesto por Landry $^{(14)}$ (Tabla 1). La evaluación tomográfica se realizó al tercer mes mediante TCCB (Point 3D Combi 500). El análisis tomográfico se avaluó mediante el software Real Sacan Versión 2.0 donde se determinó la densidad ósea de la zona de


Figura 2. Planeamiento e intervención quirúrgica. Radiografías panorámicas con terceras molares mandibulares con similitud en complejidad para las exodoncias (a y d). Lecho quirúrgico de alveolo posexodoncia (b). Preparado de PRP en estado gel (c y e). Aplicación de PRP sobre e alveolo posexodoncia (f).

\begin{tabular}{|c|c|c|c|c|c|c|}
\hline \multicolumn{7}{|c|}{ Tabla 1} \\
\hline \multicolumn{7}{|c|}{ Criterio de cicratizción de herida propuesto por Landry ${ }^{(14)}$} \\
\hline \multirow{7}{*}{$\frac{\frac{0}{\tilde{w}}}{\frac{w}{\frac{6}{v}}}$} & & & & PUNTUACIÓN & & \\
\hline & & 1 & 2 & 3 & 4 & 5 \\
\hline & $\begin{array}{l}\text { Color de } \\
\text { tejido }\end{array}$ & $>50 \%$ rojo & $>50 \%$ rojo & $\begin{array}{c}>25 \%<50 \% \\
\text { rojo }\end{array}$ & $<25 \%$ rojo & $\begin{array}{c}100 \% \\
\text { rosado }\end{array}$ \\
\hline & $\begin{array}{l}\text { Respuesta a } \\
\text { la palpación }\end{array}$ & Sangrante & Sangrante & $\begin{array}{c}\text { No } \\
\text { sangrante }\end{array}$ & $\begin{array}{c}\text { No } \\
\text { sangrante }\end{array}$ & $\begin{array}{c}\text { No } \\
\text { sangrante }\end{array}$ \\
\hline & $\begin{array}{c}\text { Tejido de } \\
\text { granulación }\end{array}$ & Presente & Presente & Ausente & Ausente & Ausente \\
\hline & $\begin{array}{l}\text { Margen de } \\
\text { incisión }\end{array}$ & $\begin{array}{l}\text { No } \\
\text { epitelizado, } \\
\text { pérdida de } \\
\text { epitelio fuera } \\
\text { del margen }\end{array}$ & $\begin{array}{c}\text { No } \\
\text { epitelizado, } \\
\text { con tejido } \\
\text { conectivo } \\
\text { expuesto }\end{array}$ & $\begin{array}{c}\text { No } \\
\text { exposición } \\
\text { de tejido } \\
\text { conectivo }\end{array}$ & $\begin{array}{c}\text { No } \\
\text { exposición } \\
\text { de tejido } \\
\text { conectivo }\end{array}$ & $\begin{array}{c}\text { No } \\
\text { exposición } \\
\text { de tejido } \\
\text { conectivo }\end{array}$ \\
\hline & Supuración & Presente & Ausente & Ausente & Ausente & Ausente \\
\hline
\end{tabular}


cicatrización de los alveolos posexodoncia. El análisis constó en colocar un implante dental virtual en la misma orientación de las piezas dentales extraídas; el software analizó la densidad ósea en Unidades Hounsfield (HU) dentro del implante y alrededor de éste (outside and inside the implant) (Figura 3). diferencia significativa en todos los casos favorable con la aplicación del PRP $(\mathrm{p}<0,05)$. El nivel de curación de herida al tercer día no mostró diferencia con la aplicación del PRP ( $p>0,05)$; sin embargo a la primera y segunda semana evidenció mejora en relación al grupo control $(\mathrm{P}<0,05)$ (Tabla 2$)$.


C
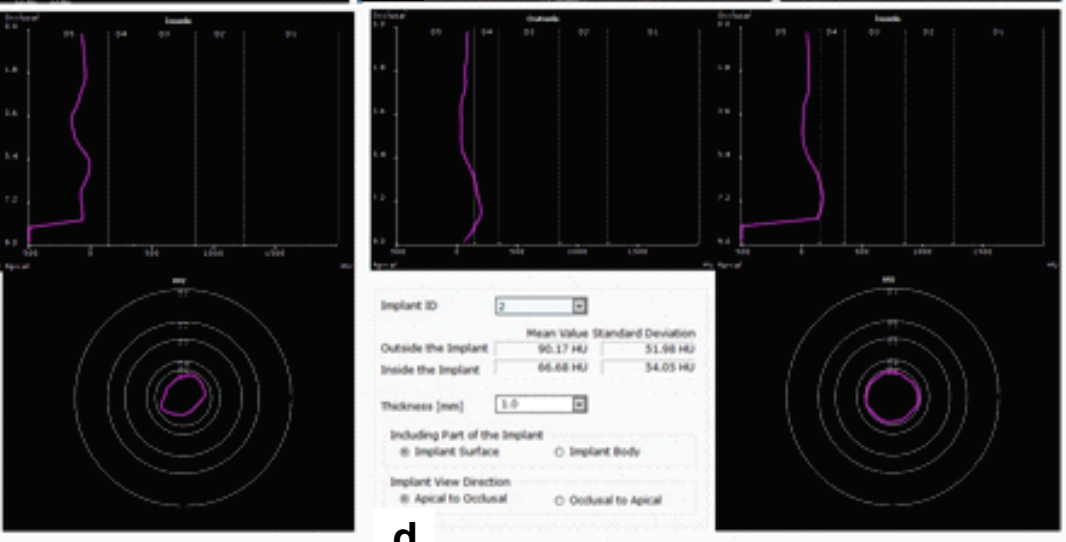

Figura 3. Software Real Scan 2.0, análisis de densidad ósea mediante la ubicación de un implante virtual en la zona de cicatrización Orientación espacial en corte coronal, sagital y axial de zona de cicatrización (a). Colocación del implante virtual en zona de cicatrización según orientación espacial de pieza dentaria extraída (b). Medición de la densidad ósea dentro y fuera del implante virtual; la medición negativa o de valor cercano a cero indica ausencia de grado de densidad ósea, los valores superior a cero indican algún grado de densidad (cyd).

Los datos del dolor según la EVA se analizaron mediante la prueba estadística de comparación de medias para muestras relacionadas según su normalidad ( $\mathrm{T}$ de Student/ Wilcoxon); la densidad ósea se comparó mediante la prueba estadística para muestras relacionadas T de Student. Todos los datos fueron analizados por el software estadístico SPSS v. 19.0 (SPSS Inc., Chicago, IL, USA)

\section{Resultados}

Ser realizó un total de 40 exodoncias en 20 pacientes, conformado por 7 personas de sexo femenino y 13 del sexo masculino, con una edad media de $22 \pm 3$ años e IMC de 23,46 $\pm 2,7$. La evaluación del dolor posoperatorio mediante la EVA al tercer día, primera semana y segunda semana mostraron una
La densidad ósea evaluada mediante TCCB evidenció que con la aplicación del PRP sobre el alveolo posexodoncia a los tres meses, la zona de cicatrización muestra mejor grado de densidad ósea que con el grupo control siendo esta diferencia significativa $(p<0,05)$, como muestra la tabla 3 .

\section{Discusión}

El uso del PRP como un biomaterial prometedor sobre la regeneración de tejidos es aún controversial, sobre todo, por el protocolo usado y los métodos de activación ${ }^{(13)}$; sin embargo, su uso extendido ha mostrado evidencia clínica satisfactoria con su aplicación en diversas especialidades médicoodontológicas $^{(7,15,16)}$. Rodríguez y col., enfatiza que la aplicación del PRP sobre un lecho quirúrgico mejora la 


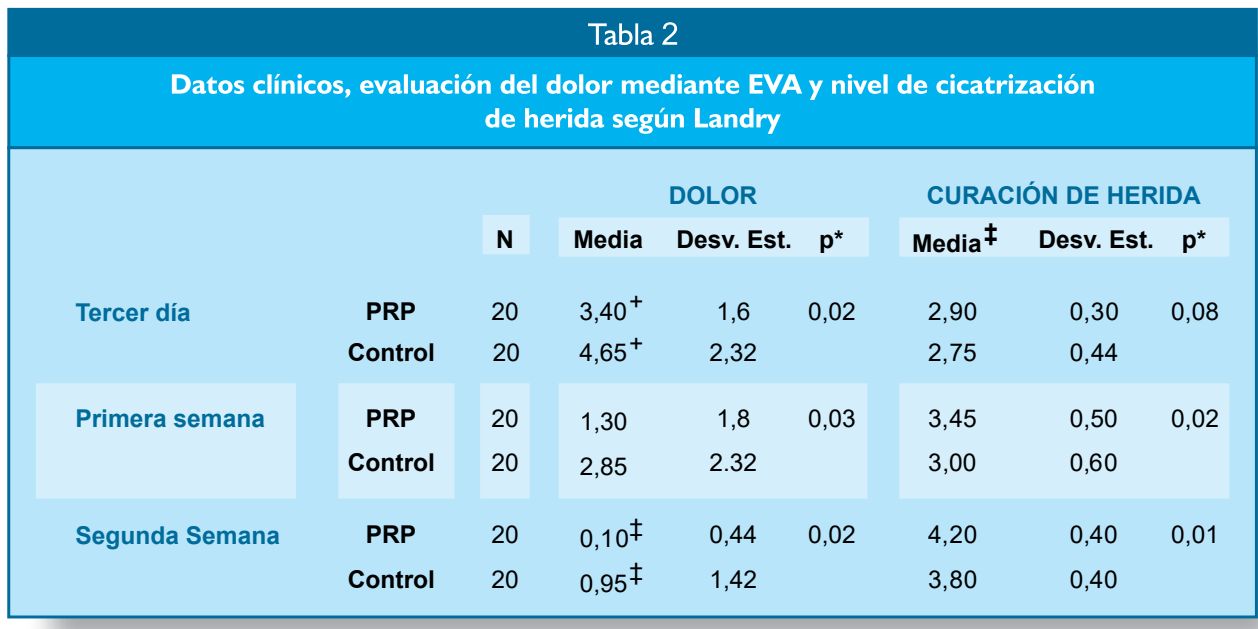

\footnotetext{
${ }^{*} p=$ Nivel de significancia estadística con IC al 95\%

+ Prueba de Shapiro Wilk $p>0,05$; prueba estadística: T de Student

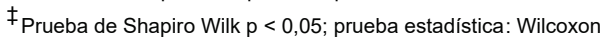

\begin{tabular}{|ccccccc|}
\hline \multicolumn{7}{c|}{ Tabla 3} \\
\hline Evaluación de la densidad ósea mediante TCCB en unidades Hounsfield (HU) \\
\hline \multirow{2}{*}{ Densidad ósea } & & $\mathbf{N}^{*}$ & Media $^{+}$ & Desv. Est. & $\mathrm{p}^{\ddagger}$ \\
Fuera de implante virtual & PRP & 19 & 77,74 & 85,25 & 0.04 \\
& Control & 19 & 40,06 & 85,38 & \\
Dentro del implante virtual & PRP & 19 & 72,14 & 80,68 & 0.03 \\
& Control & 19 & 35,59 & 94,26 & \\
\hline
\end{tabular}

${ }^{*} \mathrm{~N}$ inicial $=20$ pacientes $\mathrm{N}^{*}=19$ pacientes ( 1 paciente perdido - excluido del análisis)

+ Prueba de Shapiro Wilk $\mathrm{p}>0,05$; prueba aplicada: T de Student

$\ddagger_{p}=$ Nivel de significancia estadística con IC al 95\%

aceleración de la cicatrización, evidenciado en la disminución del dolor e indicadores de inflamación ${ }^{(7)}$. Albanese concluye que la cicatrización de tejido blando posexodoncia mejora notablemente con la aplicación del $\mathrm{PRP}^{(17)}$. Asimismo, en nuestra investigación podemos afirmar que la aplicación del PRP mejora notablemente la evolución posoperatoria con la disminución del dolor y aceleración en la cicatrización del tejido blando, puesto que existe diferencia estadísticamente significativa en comparación con el grupo control, así lo demuestran también Alissa y col. ${ }^{(18)}$, Ogundipe y col. ${ }^{(1)}$, Vivek y col. ${ }^{(19)}$.

En relación a la aceleración en la cicatrización de tejido blando Viveck ${ }^{(19)}$ y Dutta ${ }^{(14)}$ se basan en el nivel de curación de herida propuesto por Landry, al igual que en nuestra investigación. Los resultados obtenidos difieren con la significancia en los momentos evaluados; también establece diferencia significativa desde el tercer día de evaluación con puntuación de 2,5 y 1,8 para el grupo experimental y control respectivamente; en nuestro caso de 2,9 y 2,75 para el grupo experimental y control respectivamente. Asimismo, Vivek ${ }^{(19)}$ evalúa desde el primer día del posoperatorio sin diferencia significativa; pero sí, al tercer y séptimo día.

Algunas investigaciones ${ }^{(1,20.21)}$ evalúan la aceleración de la cicatrización del tejido blando mediante la existencia o no de dehiscencia de la herida, midiendo el grado de inflamación, limitación de la apertura bucal y ganancia de inserción clínica de la pieza adyacente; para todos los casos con resultados favorables con la aplicación del PRP.

La evaluación tomográfica de la densidad ósea mostró resultados favorables con la aplicación del PRR que con la no aplicación; sin embargo, resalta la limitante sobre los valores promedios obtenidos $(77,74 \pm 85,25 \mathrm{HU})$ que no sugieren regeneración ósea ${ }^{(22)}$. Anitua ${ }^{(5)}$ en su estudio con exodoncias de 
no terceros molares obtiene valores superiores de densidad ósea medidas con tomografía TCCB con vales de densidad ósea de $450 \pm 106,7$ y $318,2 \pm 113 \mathrm{HU}$ para el grupo experimental y control, respectivamente. Se deduce que difiere con nuestro estudio puesto que la evaluación tomográfica fue posterior a los 4 meses de la intervención, no fueron terceros molares y el modo de medición no fueron en las mismas circunstancias. Además en su estudio analiza el volumen del alveolo regenerado (hasta $75 \%$ superior de la medición base) consiguiendo mejor evidencia para la regeneración ósea del alveolo posexodoncia; evidencia conseguida también por Rutkowski $^{(23)}$ y Cheah $^{(24)}$, pero, en base a medidas lineales, ambos casos favorables con la aplicación del PRP.

El estudio presenta limitantes en el modo de medición de la densidad ósea, puesto que es un método novedoso; las mediciones se realizan de manera subjetiva al depender de la colocación de un implante virtual en la zona de cicatrización, pero con parámetros establecidos en las mediciones. Esta limitante también es evidenciada en estudios que miden la densidad ósea mediante la apreciación de radiografías, ya sea mediante una valoración subjetiva o la utilidad de algún software $^{(3,10.25)}$.

La evidencia reciente ${ }^{(9,26)}$ refiere que el uso del PRP sobre alveolos posexodoncia es aún cuestionable por la falta de estudios clínicos aleatorizado estructuralmente diseñados; debido a ello, esta propuesta pone en énfasis que el estudio fue realizado a boca partida (grupo control y experimental en un mismo paciente), además de proponer un protocolo con parámetros claros y reproducibles.

\section{Conclusiones}

La aplicación del PRP posee mejor efecto clínico sobre el alveolo posexodoncia disminuyendo el dolor posoperatorio y acelerando la cicatrización de tejido blando. Además, en este estudio se demuestra que con la TCCB existe mayor nivel de densidad ósea en comparación con la no aplicación del PRP. Sin embargo, los resultados no sugieren un valor de densidad ósea concluyente a regeneración.

\section{Agradecimientos}

Los autores agradecen al cirujano Bucal y Maxilofacial Andy Sotelo Ortiz por la ejecución principal de las intervenciones quirúrgicas. A los cirujanos Bucal Maxilofacial David Roldan Farfán, Janie Vásquez Navarro, Fernando Díaz Príncipe y Nelly Carrasco Ortega por su apoyo complementario en la ejecución de intervenciones quirúrgicas. Al Dr. Manuel Arrascue Dulanto como coordinador informante de la Fundación Instituto Hipólito Unanue, financiador de la investigación. Al Dr. Jaime Dexcaleaux Dulanto, Blga. Margarita Eugenia Velásquez, Dr. Renato Castro Monzón (cirujano plástico) por su colaboración en la estandarización del protocolo.
Referencias bibliográficas

1. Ogundipe OK, Ugboko VI, Owotade FJ. Can autologous platelet-rich plasma gel enhance healing after surgical extraction of mandibular third molars? J Oral Maxillofac Surg [Internet]. 2011[citado 01/04/2015];69(9):5-10. Disponible en: https://www.ncbi.nlm.nih.gov/pubmed/21550158

2. Hupp JR, Ellis III E, Tucker MR. Cirugía oral y maxilofacial contemporanea. Elsevier; 2010:728.

3. Gawai KT, Sobhana CR. Clinical evaluation of use of platelet rich plasma in bone healing. J Maxillofac Oral Surg [Internet]. 2015 [citado 08/04/2015];14(1):67-80. Disponible en: https://www.ncbi.nlm.nih.gov/pmc/articles/PMC4339326/

4. Anitua E, Tejero R, Alkhraisat MH, Orive G. Platelet-Rich Plasma to Improve the Bio-Functionality of Biomaterials. BioDrugs [Internet]. 2012 [citado 06/06/2016];27(2):97-111. Disponible en: http://link.springer.com/article/10.10 07/s40259-012-0004-3

5. Anitua E, Murias-Freijo A, Alkhraisat MH, Orive G. Clinical, radiographical, and histological outcomes of plasma rich in growth factors in extraction socket: a randomized controlled clinical trial. Clin Oral Investig [Internet]. 2015 [citado 15/05/2015]; 19(3):589-600. Disponible en: https:// link.springer.com/article/10.1007/s00784-014-1278-2

6. Arenaz-Búa J, Luaces-Rey R, Sironvalle-Soliva S, OteroRico A, Charro-Huerga E, Patiño-Seijas B, et al. A comparative study of platelet-rich plasma, hydroxyapatite, demineralized bone matrix and autologous bone to promote bone regeneration after mandibular impacted third molar extraction. Med Oral Patol Oral Cirugia Bucal [Internet]. 2010 [citado 01/04/2015];15(3):e483-9. Disponible en: https://www.ncbi.nlm.nih.gov/pubmed/20038911

7. Rodríguez Flores J, Gallego P, Angustias M, Torres GarcíaDenche J. Plasma rico en plaquetas: fundamentos biológicos y aplicaciones en cirugía maxilofacial y estética facial. Rev Esp Cir Oral Maxilofac [Internet]. 2012 [citado 04/02/2017]; 34(1):8-17. Disponible en: http://scielo.isciii.es/scielo.php? script $=$ sci_arttext\&pid=S1130-05582012000100002

8. Marx RE. Platelet-rich plasma: evidence to support its use. J Oral Maxillofac Surg [Internet]. 2004 [citado 27/08/2015]; 62(4): 489-96. Disponible en: http://www.sciencedirect.com/ science/article/pii/S0278239103012722

9. Barona-Dorado C, González-Regueiro I, Martín-Ares M, Arias-Irimia O, Martínez-González JM. Efficacy of platelet- 
rich plasma applied to post-extraction retained lower third molar alveoli. A systematic review. Med Oral Patol Oral Cirugia Bucal [Internet]. 2014 [citado 01/04/2015];19(2):1428. Disponible en: https://www.ncbi.nlm.nih.gov/pubmed/ 24316707

10. Antonello GDM, Torres Do Couto R, Giongo CC, Corrêa MB, Chagas Júnior OL, Lemes CHJ. Evaluation of the effects of the use of platelet-rich plasma (PRP) on alveolar bone repair following extraction of impacted third molars: Prospective study. J Cranio-Maxillofac Surg [Internet]. 2013 [citado 04/04/2017];41(4):70-5. Disponible en: https://www. ncbi.nlm.nih.gov/pubmed/23352081

11. Eskan MA, Greenwell H, Hill M, Morton D, Vidal R, Shumway B, et al. Platelet-rich plasma-assisted guided bone regeneration for ridge augmentation: a randomized, controlled clinical trial. J Periodontol [Internet]. 2014 [citado 08/04/2015]; 85(5):661-8. Disponible en: https://www.ncbi. nlm.nih.gov/pubmed/23895252

12. Araki J, Jona M, Eto H, Aoi N, Kato H, Suga H, et al. Optimized Preparation Method of Platelet-Concentrated Plasma and Noncoagulating Platelet-Derived Factor Concentrates: Maximization of Platelet Concentration and Removal of Fibrinogen. Tissue Eng Part C Methods [Internet]. 2012 [citado 04/04/2017];18(3):176-85. Disponible en: https://www.ncbi.nlm.nih.gov/pubmed/21951067

13. Dhurat R, Sukesh M. Principles and Methods of Preparation of Platelet-Rich Plasma: A Review and Author's Perspective. J Cutan Aesthetic Surg [Internet]. 2014 [citado 04/04/2017]; 7(4):189-97. Disponible en: https://www.ncbi.nlm.nih. gov/pubmed $/ 25722595$

14. Dutta SR, Singh P, Passi D, Patter P. Mandibular Third Molar Extraction Wound Healing With and Without Platelet Rich Plasma: A Comparative Prospective Study. J Maxillofac Oral Surg [Internet]. 2015 [citado 06/04/2015];14(3):808-815. Disponible en: http://link.springer.com/article/10.1007/ s12663-014-0738-1

15. Anitua E, Tejero R, Alkhraisat MH, Orive G. Platelet-rich plasma to improve the bio-functionality of biomaterials. BioDrugs Clin Immunother Biopharm Gene Ther [Internet]. 2013[citado 06/06/2016];27(2):97-111. Disponible en: http://link.springer.com/article/10.1007/s40259-012-0004-3

16. González Lagunas J. Plasma rico en plaquetas. Rev Esp Cir Oral Maxilofac [Internet]. 2006 [citado 27/05/2015]; 28(2):8999. Disponible en: http://scielo.isciii.es/scielo..php? s c r i p t $=$ s c i a b s t r a c t \& p i d = S 1130 $005582006000200001 \& \operatorname{lng}=$ es\&nrm=iso\&tlng=es

17. Albanese A, Licata ME, Polizzi B, Campisi G. Platelet-rich plasma (PRP) in dental and oral surgery: from the wound healing to bone regeneration. Immun Ageing A [Internet]. 2013[citado 27/05/2015];10:23. Disponible en: https://www. ncbi.nlm.nih.gov/pubmed/23763951

18. Alissa R, Esposito M, Horner K, Oliver R. The influence of platelet-rich plasma on the healing of extraction sockets: an explorative randomised clinical trial. Eur J Oral Implantol [Internet]. 2010[citado 17/05/2015];3(2):121-34. Disponible en: https://www.ncbi.nlm.nih.gov/pubmed/20623037

19. Vivek GK, Sripathi Rao BH. Potential for osseous regeneration of platelet rich plasma: a comparitive study in mandibular third molar sockets. J Maxillofac Oral Surg [Internet]. 2009[citado 07/02/2016];8(4):308-11. Disponible en: https://www.ncbi.nlm.nih.gov/pmc/articles/PMC3454099/

20. Dutta SR, Passi $D$, Singh $P$, Sharma $S$, Singh M, Srivastava D. A randomized comparative prospective study of plateletrich plasma, platelet-rich fibrin, and hydroxyapatite as a graft material for mandibular third molar extraction socket healing. Natl J Maxillofac Surg. junio de 2016;7(1):45-51.

21. Kaul RP, Godhi SS, Singh A. Autologous Platelet Rich Plasma After Third Molar Surgery: A Comparative Study. J Maxillofac Oral Surg [Internet]. junio de 2012 [citado 07/02/2016]; 11(2):200-5. Disponible en: http://www.ncbi.nlm.nih. gov/pmc/articles/PMC3386415/

22. Andersson L, Kahnberg K-E, Pogrel T. Tratado de Cirugía Oral Maxilofacial y Prótesis para Tejidos Blandos. Vol. 1. Amolca; 2015;1274.

23. Rutkowski JL, Johnson DA, Radio NM, Fennell JW. Platelet Rich Plasma to Facilitate Wound Healing Following Tooth Extraction. J Oral Implantol [Internet]. 2010 [citado 15/05/2015]; 36(1):11-23. Disponible en: https://www.ncbi. nlm.nih.gov/pubmed/20218866

24. Cheah CW, Vaithilingam RD, Siar CH, Swaminathan D, Hornbuckle GC. Histologic, histomorphometric, and conebeam computerized tomography analyses of calcium sulfate and platelet-rich plasma in socket preservation: a pilot study. Implant Dent [Internet]. 2014 [citado 14/05/2015]; 23(5):593601. Disponible en: https://www.ncbi.nlm.nih.gov/pubmed/ 25192162

25. Hiremath H, Motiwala T, Jain P, Kulkarni S. Use of secondgeneration platelet concentrate (platelet-rich fibrin) and hydroxyapatite in the management of large periapical inflammatory lesion: a computed tomography scan analysis. Indian J Dent Res Off Publ Indian Soc Dent Res [Internet]. 2014 [citado 14/05/2015];25(4):517-20. Disponible en: https://www.ncbi.nlm.nih.gov/pubmed/25307920

26. Plachokova AS, Nikolidakis D, Mulder J, Jansen JA, Creugers NHJ. Effect of platelet-rich plasma on bone regeneration in dentistry: a systematic review. Clin Oral Implants Res [Internet]. 2008 [citado 14/05/2015];19(6):53945. Disponible en: https://onlinelibrary.wiley.com/doi/abs/ 10.1111/j.1600-0501.2008.01525.x

Contribución de autoría: DQR diseño del estudio, investigador principal de la investigación, redacción del artículo. TEC diseño del estudio, asesora en el soporte estadístico y redacción del artículo. MGP diseño del estudio, asesora en la ejecución de las intervenciones quirúrgicas y redacción del artículo. DVC diseño del estudio, estandarización del protocolo del biomaterial, redacción del artículo. SCG diseño del estudio, asesor en la evaluación radiológica y redacción del artículo. SGP diseño del estudio, asesor principal de la investigación, redacción del artículo.

Conflicto de interés: Los autores no tienen conflictos de interés con la publicación de este trabajo.

Financiamiento: La investigación fue financiada por la Fundación Instituto Hipólito Unanue (FIHU)

Citar como: Quincho Rosales DA., y cols. Efecto del plasma rico en plaquetas en la regeneración ósea posexodoncia del tercer molar impactado evaluado a través de tomografía computarizada Cone Beam. Diagnóstico(Lima). 2021;60(2):71-78.

DOI: 10.33734 /diagnostico.v60i2.283

Correspondencia: Dilmer Armando Quincho Rosales. Correo electrónico: dilarmy@gmail.com 